doi: $10.13108 / 2020-12-3-22$

\title{
RECOVERING OF TWO-POINT BOUNDARY CONDITIONS BY FINITE SET OF EIGENVALUES OF BOUNDARY VALUE PROBLEMS FOR HIGHER ORDER DIFFERENTIAL EQUATIONS
}

\author{
B.E. KANGUZHIN
}

\begin{abstract}
The recovering of boundary conditions for higher order differential equations by some set of spectra is difficult because of two facts. First, opposite to second order differential equations, there are not triangle transformation operators for higher order differential equations. Second, non-separable boundary conditions give additional analytic problems while recovering them by the set of spectra. In the present work we provide a new way of normalizing boundary conditions, which is adapted for further recovering by some set of spectra of boundary value problems. In other words, before posing the issue by which data the boundary conditions can be recovered, one should first reduce them to a canonical form. Then, basing on an assumed canonical form, a system of boundary value problems is to be chosen and by the their spectra boundary conditions are to recovered.

We propose an algorithm of recovering two-point boundary conditions in a boundary value problem for higher order differential equations. As an additional information, a finite set of eigenvalues of special boundary value problems serve. According the terminology by V.A. Sadovnichii, such problems are called canonical problems.
\end{abstract}

Keywords: boundary conditions, boundary value problems, canonical problems.

Mathematics Subject Classification: 34B05, 47A10

\section{INTRODUCTION}

Recovering of ordinary differential equations and associated boundary conditions by the set of eigenvalues was considered in works [1]-44. In monograph [5], the methods for recovering differential operators on the segments were systematized. In the case of non-separable boundary conditions, the authors of [5] propose a method of canonical problems. According their approach, for each boundary value problem with non-separable boundary conditions for a differential equation, one should find a set of canonical problems, by the spectra of which the original operator can be recovered. However, in our opinion, the method of finding canonical problems requires a certain systematization. In the present work the process of finding canonical problems is systematized in a certain extent.

Generally speaking, the process of recovering boundary conditions and differential equation consists in two steps. At the first step, by some spectral data, one should recover the coefficients of the differential equation, and then at the second step the coefficients of the boundary conditions are found. We note that now the methods for recovering the coefficients in the differential equation are developed quite well; at the same time, some coefficients in the boundary

B.E. Kanguzhin, ReCOVERING of TWO-POINT BOUndARY CONDITIONS BY FINITE SET OF EIGENVALUES OF BOUNDARY VALUE PROBLEMS FOR HIGHER ORDER DIFFERENTIAL EQUATIONS.

CKAnguzhin B.E. 2020.

The work is supported by the Science Committee of Ministry of Education and Science of the Republic of Kazakhstan (grant AP05131292).

Submitted January 15, 2020. 
conditions can be recovered. In the general case, the recovering of remaining coefficients in the boundary conditions is an unavoidable step. In monograph [5], the aforementioned approach of two-step recovering of a differential operator is promoted.

In the present work we suppose that the first step of recovering the coefficients of a differential equation is over and it remains to recover the coefficients of the boundary conditions in a boundary value problem for a given differential equation by some set of the eigenvalues of some canonical problems. First, one needs to specify the set of canonical problems. Second, how many and which eigenvalues of the chosen canonical problems determine uniquely the boundary conditions in the original boundary value problems?

\section{ORIGINAL TWO-POINT BOUNDARY VALUE PROBLEM}

In the space $L_{2}(0,1)$ we consider the eigenvalue problem

$$
\begin{aligned}
& d(y) \equiv y^{(n)}(x)+\sum_{k=0}^{n-2} p_{k}(x) y^{(k)}(x)=\lambda y(x), \quad 0<x<1 \\
& V_{j}(y) \equiv \sum_{s=1}^{n}\left(\alpha_{j s} y^{(s-1)}(0)+\beta_{j s} y^{(s-1)}(1)\right)=0, \quad j=1, \ldots, n
\end{aligned}
$$

where $p_{k}(x)$ are sufficiently smooth coefficients of a differential equation, $\alpha_{j s}, \beta_{j s}$ are scalar coefficients of boundary conditions. We assume that $\lambda=0$ is not an eigenvalue of problem (2.1) and (2.2). A direct problem of spectral analysis reads as follows: given a differential expression $d(\cdot)$ and a set of boundary forms $\left\{V_{j}(\cdot)\right\}$, study the spectrum and the properties of the systems of root functions of problem (2.1)-(2.2). According our assumption, the resolvent set of problem (2.1)-2.2) is non-empty, and hence, the spectrum of the original problem consists of countably many eigenvalues [6]. While solving the direct problem, one should first form the set of boundary conditions (2.1)-(2.2). For instance [6], if conditions (2.2) are equivalent to boundary conditions regular in the Birkhoff sense, then the system of root functions of problem 2.1) - 2.2 forms a complete system in $L_{2}(0,1)$.

In the present work we study an inverse problem: given differential equation (2.1) and some set of the eigenvalues of canonical problems, recover uniquely the coefficients of boundary conditions (2.2). In what follows we shall specify how to find canonical problems. Before we choose canonical problems, we need to normalize boundary conditions in a special way.

It turns out that solving of direct and inverse problems is to be begun by normalizing the set of boundary conditions (2.2). In other words, set of canonical boundary conditions (2.2) should be first reduced to some equivalent canonical form.

\section{EQUiVALENT CANONICAL BOUNDARY CONDITIONS}

In this section we describe how to normalize the set of two-points boundary conditions and specify then the formulation of the inverse problem.

We introduce a fundamental system of solutions $\left\{y_{i}(x)\right\}$ of a homogeneous equation $d(y)=0$ with standard Cauchy conditions at zero: $y_{i}^{(s-1)}(0)=\delta_{i s}$. Hereinafter the symbol $\delta_{i s}$ stands for the Kronecker delta. The determinant

$$
\left|\begin{array}{cccc}
y_{1}(t) & y_{2}(t) & \cdots & y_{n}(t) \\
\vdots & \vdots & \ddots & \vdots \\
y_{1}^{(n-2)}(t) & y_{2}^{(n-2)}(t) & \cdots & y_{n}^{(n-2)}(t) \\
y_{1}(x) & y_{2}(x) & \cdots & y_{n}(x)
\end{array}\right|
$$


is denoted by $g(x, t)$. It is known [6] that a function $u_{0}(x)$ defined by the formula

$$
u_{0}(x)=\int_{0}^{x} g(x, t) f(t) d t
$$

is a solution to an inhomogeneous Cauchy problem with homogeneous conditions at zero:

$$
d\left(u_{0}\right)=f(x), \quad u_{0}^{(s-1)}(0)=0 .
$$

We also note that the following relations hold for $s=1, \ldots, n$ :

$$
u_{0}^{(s-1)}(x)=\int_{0}^{x} \frac{\partial^{s-1}}{\partial x^{s-1}} g(x, t) f(t) d t,
$$

Now we are in position to formulate a statement.

Lemma 3.1. For each $f$ in $L_{2}(0,1)$, an inhomogeneous equation $d(u)=f(x)$ possesses the unique solution satisfying conditions (2.2). This solution satisfies the representation:

$$
u(x)=u_{0}(x)-\sum_{s=1}^{n} \varphi_{s}(x) V_{s}\left(u_{0}\right)
$$

Here $\left\{\varphi_{s}(x)\right\}$ is the system of solutions to the homogeneous equation $d(y)=0$ with conditions $V_{j}\left(\varphi_{s}\right)=\delta_{j s}, j=1, \ldots, n$.

Lemma 3.1 can be confirmed straightforwardly. The uniqueness is implied by the assumption that $\lambda=0$ is not an eigenvalue of problem (2.1) and (2.2). Let us find the values of boundary forms $V_{j}\left(u_{0}\right)$. Recalling the properties of $u_{0}(x)$, we have:

$$
V_{j}\left(u_{0}\right)=\left.\sum_{s=1}^{n} \beta_{j s} \int_{0}^{1} \frac{\partial^{s-1}}{\partial x^{s-1}} g(x, t)\right|_{x=1} f(t) d t .
$$

By representation (3.1) we obtain the identity

$$
u(x)+\sum_{j=1}^{n} \varphi_{j}(x) V_{j}\left(u_{0}\right)=u_{0}(x) .
$$

Therefore, the function $u_{0}(x)$ satisfies the representation

$$
u_{0}(x)=u(x)+\left.\sum_{j=1}^{n} \varphi_{j}(x) \sum_{s=1}^{n} \beta_{j s} \int_{0}^{1} \frac{\partial^{s-1}}{\partial x^{s-1}} g(x, t)\right|_{x=1} f(t) d t .
$$

The latter identity can be rewritten as

$$
u_{0}(x)=u(x)+\int_{0}^{1} \theta(x, t) f(t) d t
$$

where

$$
\theta(x, t)=\left.\sum_{j=1}^{n} \varphi_{j}(x) \sum_{s=1}^{n} \beta_{j s} \int_{0}^{1} \frac{\partial^{s-1}}{\partial x^{s-1}} g(x, t)\right|_{x=1} .
$$


Since $u_{0}^{(s-1)}(0)=0$ and $d(u)=f(x)$, the relations

$$
u^{(s-1)}(0)+\int_{0}^{1} \rho_{s}(t) d(u) d t=0, \quad s=1, \ldots, n,
$$

hold, where

$$
\rho_{s}(t)=\left.\frac{\partial^{s-1}}{\partial x^{s-1}} \theta(x, t)\right|_{x=0} .
$$

Now we introduce the set of boundary forms by the formulae

$$
W_{s}(u)=u^{(s-1)}(0)+\int_{0}^{1} \rho_{s}(t) d(u) d t, \quad s=1, \ldots, n .
$$

Hence, we have proved the following statement.

Theorem 3.1. Set of boundary conditions (2.2) is equivalent to the following boundary conditions:

$$
W_{s}(u) \equiv u^{(s-1)}(0)+\int_{0}^{1} \rho_{s}(t) d(u) d t=0, \quad s=1, \ldots, n .
$$

We call boundary conditions defined by Theorem 3.1 canonical boundary conditions or normalized boundary conditions. Thus, the set of boundary form $\left\{V_{j}(\cdot)\right\}$ is equivalent to the canonical set of boundary forms $\left\{W_{j}(\cdot)\right\}$. This is why, instead of recovering boundary conditions $\left\{V_{j}(\cdot)\right\}$, we shall recover boundary conditions $\left\{W_{j}(\cdot)\right\}$.

\section{Choice of CANONiCAL PROBlems AND SPECIFIED FORMULATION OF INVERSE PROBLEM}

In this section we provide a way of choosing canonical problems, the spectra of which determine uniquely the boundary conditions in the original problem or equivalent boundary conditions. In fact, for determining the boundary coefficients not entire spectrum is employed but only its finite part.

The number of auxiliary canonical problems is equal to the order of differential equation 2.1). That is, we construct $n$ canonical problems. As a first canonical problem, the following one serves:

$$
\begin{aligned}
& d(y) \equiv y^{(n)}(x)+\sum_{k=0}^{n-2} p_{k}(x) y^{(k)}(x)=\lambda y(x), \quad 0<x<1, \\
& W_{1}(y)=0, y^{(k-1)}(0)=0, \quad k=2, \ldots, n .
\end{aligned}
$$

The second canonical problem reads as

$$
\begin{aligned}
& d(y) \equiv y^{(n)}(x)+\sum_{k=0}^{n-2} p_{k}(x) y^{(k)}(x)=\lambda y(x), \quad 0<x<1, \\
& W_{1}(y)=0, W_{2}(y)=0, y^{(k-1)}(0)=0, \quad k=3, \ldots, n .
\end{aligned}
$$

In the same we choose the third, the fourth, $\ldots,(n-1)$ th canonical problems. As $n$th canonical problem we choose the following problem:

$$
\begin{aligned}
& d(y) \equiv y^{(n)}(x)+\sum_{k=0}^{n-2} p_{k}(x) y^{(k)}(x)=\lambda y(x), \quad 0<x<1, \\
& W_{k}(y)=0, k=1, \ldots, n-1, y^{(n-1)}(0)=0 .
\end{aligned}
$$


Now we are in position to provide a specified formulation of the inverse problem.

The first inverse problem: given differential equation (2.1) and the spectrum of the first canonical problem, recover uniquely the first boundary function $\rho_{1}(t)$. The second inverse problem: by the given differential equation (2.1), the found boundary function $\rho_{1}(t)$ and the spectrum of the second canonical problem, recover uniquely the second boundary function $\rho_{1}(t)$. In the same the third, the fourth,..., $(n-1)$ th inverse problems are formulated. The $n$th inverse problem reads as: by the given differential equation (2.1), the found boundary functions $\rho_{1}(t), \ldots, \rho_{n-1}(t)$ and the spectrum of $n$th canonical problem, recover uniquely $n$th boundary function $\rho_{n}(t)$. In fact, not entire spectrum of a canonical problem is employed, but only its finite part. In the following sections we shall study in details this aspect.

\section{TheOREM ON UNIQUE RECOVERING OF BOUNDARY FUNCTIONS}

By passing from boundary conditions $(2.2)$ to equivalent canonical boundary forms, we shall prove a theorem on unique recovering of boundary conditions $\rho_{1}(t), \ldots, \rho_{n}(t)$. In what follows, the $s$ th canonical problem is called Problem $E_{s}$. Problem $E_{s}$ with the same equation (2.1), but with other parameters in boundary conditions $(2.2)$ is denoted by $\widetilde{E}_{s}$. Hereafter, if some symbol denotes an object related to Problem $E_{s}$, then the same symbol with tilde denotes a similar object for problem $\widetilde{E}_{s}$.

Theorem 5.1. Fix an integer $s$ in the set $\{1, \ldots, n\}$. Assume that the spectra of Problems $E_{s}$ and $\widetilde{E}_{s}$ coincide. If $\rho_{1}(t)=\widetilde{\rho_{1}(t)}, \ldots, \rho_{s-1}(t)=\widetilde{\rho_{s-1}(t)}$ in $L_{2}(0,1)$ and the systems of root functions of Problems $E_{s}$ and $\widetilde{E_{s}}$ are complete in $L_{2}(0,1)$, then $\rho_{s}(t)=\widetilde{\rho_{s}(t)}$ in $L_{2}(0,1)$.

Remark 5.1. In the case of two-points boundary value problems (2.1)-(2.2), the boundary functions $\rho_{1}(t), \ldots, \rho_{n}(t)$ are smooth enough. Moreover, each of them solves a homogeneous equation $d^{+}(y)=0$, where $d^{+}(\cdot)$ is a formally adjoint differential expression. Therefore, boundary functions $\rho_{1}(t), \ldots, \rho_{n}(t)$ belong to a finite-dimensional and their recovering is reduced to determining finitely many constants. This is why, the completeness of the system of the root functions of Problems $E_{s}$ and $\widetilde{E}_{s}$ is superfluous. Howerver, Theorem 2 holds for the boundary functions $\rho_{1}(t), \ldots, \rho_{n}(t)$ in $L_{2}(0,1)$.

Proof of Theorem 5.1. Case $s=1$. We introduce a fundamental system of solutions $\left\{y_{i}(x)\right\}$ of the homogeneous equation $d(y)=\lambda y(x)$ with standard Cauchy conditions at zero $y_{i}^{(s-1)}(0)=$ $\delta_{i s}$. Let $\lambda=\lambda^{(1)}$ be an arbitrary eigenvalue of problem $E_{1}$. Then $y_{1}\left(x, \lambda^{(1)}\right)$ is an eigenfunction of Problem $E_{1}$ associated with the eigenvalue $\lambda^{(1)}$. The first boundary condition in Problem $E_{1}$ becomes

$$
y_{1}\left(0, \lambda^{(1)}\right)+\int_{0}^{1} \rho_{1}(t) d\left(y_{1}\right) d t=0
$$

Since

$$
d\left(y_{1}\right)=\lambda^{(1)} y_{1}\left(x, \lambda^{(1)}\right)
$$

then

$$
\int_{0}^{1} \rho_{1}(t) y_{1}\left(t, \lambda^{(1)}\right) d t=-\frac{1}{\lambda^{(1)}} .
$$

Therefore, the eigenvalues of Problem $E_{1}$ determine the Fourier coefficients of the function $\rho_{1}(t)$ over the system of root functions of the problem adjoint for Problem $E_{1}$. Since the system of root functions of Problem $E_{1}$ is complete in the space $L_{2}(0,1)$, then the system of the root functions of the adjoint functions is also complete in $L_{2}(0,1)$. Thus, if the spectra of Problems $E_{s}$ and $\widetilde{E}_{s}$ 
coincide, the same is true the Fourier coefficients of the functions $\rho_{1}(t)$ and $\widetilde{\rho_{1}(t)}$ over the same complete system in the space $L_{2}(0,1)$. Therefore, the functions $\rho_{1}(t)$ and $\widetilde{\rho_{1}(t)}$ coincide in the space $L_{2}(0,1)$. This proof corresponds to the case of simple eigenvalues of Problems $E_{s}$ and $\widetilde{E}_{s}$. In the case of existence of multiple eigenvalues the arguing should be modified a little.

Case $s=2$. We introduce a solution $\chi_{2}(x, \lambda)$ to homogeneous equation $d(y)=\lambda y(x)$ with conditions $\chi_{2}^{(s-1)}(0)=\delta_{2 s}, s=2, \ldots, n, W_{1}\left(\chi_{2}\right)=0$. Let $\lambda=\lambda^{(2)}$ be an arbitrary eigenvalue of problem $E_{2}$. Then $\chi_{2}\left(x, \lambda^{(2)}\right)$ is an eigenfunction of Problem $E_{2}$ associated with the eigenvalue $\lambda^{(2)}$. The second boundary condition in Problem $E_{2}$ becomes

$$
\chi_{2}^{(1)}\left(0, \lambda^{(2)}\right)+\int_{0}^{1} \rho_{2}(t) d\left(\chi_{2}\right) d t=0 .
$$

Since

$$
d\left(\chi_{2}\right)=\lambda^{(2)} \chi_{2}\left(x, \lambda^{(2)}\right)
$$

then

$$
\int_{0}^{1} \rho_{2}(t) \chi_{2}\left(t, \lambda^{(2)}\right) d t=-\frac{1}{\lambda^{(2)}} .
$$

Further arguing reproduces the proof of Theorem 5.1 as $s=1$. In the case when Problems $E_{1}$ and $E_{2}$ have joint eigenvalues, minor modifications in the above arguing is needed. The proof for other values of $s$ is similar.

\section{SPECIFICATION OF UNIQUENESS THEOREM IN CASE OF TWO-POINT BOUNDARY VALUE PROBLEMS}

In the present section we specify Theorem 2 for two-point boundary value problems. In Section 3 of the present paper we have provided a relation between the coefficients $\left\{\beta_{j s}\right\}$ in boundary conditions (2.2), the coefficients $\left\{p_{k}(x)\right\}$ in differential equation (2.2) and the functions $\rho_{1}(t), \ldots, \rho_{n}(t)$. We recall that

$$
\rho_{k}(t)=\left.\sum_{j=1}^{n} \varphi_{j}^{(k-1)}(0) \sum_{s=1}^{n} \beta_{j s} \frac{\partial^{s-1}}{\partial x^{s-1}} g(x, t)\right|_{x=1} .
$$

It is known [6] that at $x=1$, the functions $\frac{\partial^{s-1}}{\partial x^{s-1}} g(x, t)$ solve the homogeneous equation $d^{+}(y)=$ 0 , where $d^{+}(\cdot)$ is the formally adjoint differential expression. Therefore, boundary functions $\rho_{1}(t), \ldots, \rho_{n}(t)$ are also solutions to the homogeneous equation $d^{+}(y)=0$.

Since the coefficients of the differential expression $d(\cdot)$ are given, then the formally adjoint expression $d^{+}(\cdot)$ is also known. We denote by $\left\{z_{i}(x)\right\}$ the fundamental solution of the homogeneous equation $d^{+}(z)=0$ with standard Cauchy conditions at zero: $z_{i}^{(s-1)}(0)=\delta_{i s}$. Let

$$
\rho_{k}(t)=c_{1} z_{1}(t)+c_{2} z_{2}(t)+\cdots+c_{n} z_{n}(t)
$$

with unknown constants $c_{1}, c_{2}, \ldots, c_{n}$. In Section 5 of the present paper, the relation between the Fourier coefficients of the boundary functions $\rho_{k}(t)$ and eigenvalues of Problem $E_{k}$ has been described. We recall that

$$
\int_{0}^{1} \rho_{k}(t) u_{k}(t) d t=-\frac{1}{\lambda^{(k)}}
$$


where $u_{k}(t)$ is an eigenfunction of Problem $E_{k}$ associated with the eigenvalue $\lambda^{(k)}$. We hence obtain a system of equations for unknown constants $c_{1}, c_{2}, \ldots, c_{n}$

$$
c_{1} \int_{0}^{1} z_{1}(t) u_{k}(t) d t+c_{2} \int_{0}^{1} z_{2}(t) u_{k}(t) d t+\cdots+c_{n} \int_{0}^{1} z_{n}(t) u_{k}(t) d=-\frac{1}{\lambda^{(k)}} .
$$

Thus, for the unique determination of unknown constants $c_{1}, c_{2}, \ldots, c_{n}$, it is sufficient to choose eigenvalues of problem $E_{k}$ so that the determinant

$$
\left|\begin{array}{ccc}
\int_{0}^{1} z_{1}(t) u_{k 1}(t) d t & \cdots & \int_{0}^{1} z_{n}(t) u_{k 1}(t) d t \\
\vdots & \ddots & \vdots \\
\int_{0}^{1} z_{1}(t) u_{k n}(t) d t & \cdots & \int_{0}^{1} z_{n}(t) u_{k n}(t) d t
\end{array}\right|
$$

is non-zero. Here $u_{k 1}(t), \ldots, u_{k n}(t)$ are the eigenfunctions of Problem $E_{k}$ associated with the chosen eigenvalues.

Lemma 6.1. Assume that Problem $E_{k}$ possesses infinitely many eigenvalues. There exist a set of eigenvalues $\left\{\lambda_{j}^{(k)}, j=j_{1}, \ldots, j_{n}\right\}$ of Problems $E_{k}$ such that the determinant

$$
A=\left|\begin{array}{ccc}
\int_{0}^{1} z_{1}(t) u_{k 1}(t) d t & \cdots & \int_{0}^{1} z_{n}(t) u_{k 1}(t) d t \\
\vdots & \ddots & \vdots \\
\int_{0}^{1} z_{1}(t) u_{k n}(t) d t & \cdots & \int_{0}^{1} z_{n}(t) u_{k n}(t) d t
\end{array}\right|
$$

is non-zero. Here $u_{k 1}(t)$ is an eigenfunction of Problem $E_{k}$ associated with the eigenvalue $\lambda_{j}^{(k)}$, $j=j_{1}$. The functions $u_{k 2}(t), \ldots, u_{k n}(t)$ have the same meaning.

Proof. We assume the opposite: for each set $\left\{\lambda_{j}^{(k)}, j=j_{1}, \ldots, j_{n}\right\}$ the determinant vanishes: $A=0$. We let $j_{1}=1, \ldots, j_{n-1}=n-1$. Since Problem $E_{k}$ possesses infinitely many eigenvalues, let $\lambda_{j}^{(k)}, j=j_{n}$, ranges over entire spectrum of problem $E_{k}$. Since $A=0$, the system of linear algebraic equations

$$
c_{1} \int_{0}^{1} z_{1}(t) u_{k j}(t) d t+c_{2} \int_{0}^{1} z_{2}(t) u_{k j}(t) d t+\cdots+c_{n} \int_{0}^{1} z_{n}(t) u_{k j}(t) d t=0, \quad j=1, \ldots, n
$$

possesses a non-zero solution. Suppose that $c_{n}=1$. Therefore, the Fourier coefficients of the function $z_{n}(t)$ are expressed linearly via the Fourier coefficients of the functions $\left\{z_{1}(x), \ldots, z_{n-1}(t)\right\}$ :

$$
\int_{0}^{1} z_{n}(t) u_{k j}(t) d t=-c_{1} \int_{0}^{1} z_{1}(t) u_{k j}(t) d t-\cdots-c_{n-1} \int_{0}^{1} z_{n-1}(t) u_{k j}(t) d t .
$$

If all Fourier coefficients of the function $z_{n}(t)$ are expressed linearly via the Fourier coefficients of the functions $\left\{z_{1}(x), \ldots, z_{n-1}(t)\right\}$, then the system of the functions $\left\{z_{1}(x), \ldots, z_{n}(t)\right\}$ is linearly dependent. The latter contradicts its choice. If $c_{1}=1$ does not hold, some modification is needed in the above arguing. Now Lemma 6.1 and Theorem 5.1 imply the desired statement. The proof is complete. 
Theorem 6.1. We fix an integer $s$ in the set $\{1, \ldots, n\}$. Let finite sets of the eigenvalues of Problems $E_{s}$ and $\widetilde{E}_{s}$ in Lemma 2 coincide. If $\rho_{1}(t)=\widetilde{\rho_{1}(t)}, \ldots, \rho_{s-1}(t)=\widetilde{\rho_{s-1}(t)}$ in $L_{2}(0,1)$, then $\rho_{s}(t)=\widehat{\rho_{s}(t)}$ in $L_{2}(0,1)$.

It was established in the proof of Theorem 5.1 that the eigenfunctions of Problems $E_{s}$ and $\widetilde{E}_{s}$ coincide if the corresponding eigenvalues do. This fact plays an essential role in the proof of Theorem 6.1. In conclusion we mention that some of our constructions can be found in works [7, 8].

\section{BIBLIOGRAPHY}

1. Z.L. Lejbenzon. An inverse problem of spectral analysis of ordinary differential operators of higher order // Trudy Mosk. Matem. Obsch. 15, 70-144 (1966). [Trans. Mosc. Math. Soc. 15, 78-163 (1966).]

2. B.M. Levitan, M.G. Gasymov. Determination of a differential equation by two of its spectra // Uspekhi Matem. Nauk. 19:2 (116), 3-63 (1964). [Russian Math. Surveys. 19:2, 1-63 (1964).]

3. V.A. Yurko. On Differential operators with nonseparated boundary conditions // Funkts. Anal. Pril. 28:4, 90-92 (1994). [Funct. Anal. Appl. 28:4, 295-297 (1994).]

4. V.A. Sadovnichii. Uniqueness of the solution to the inverse problem in the case of a second-order equation with indecomposable boundary conditions. Regularized sums of some of the eigenvalues. Factorization of the characteristic determinant// Dokl. AN SSSR. 206:2, 293-296 (1972). [Sov. Math., Dokl. 13, 1220-1223 (1972).]

5. V.A. Sadovnichii, Ya.T. Sultanaev, A.M. Akhtyamov. Inverse Sturm-Liouville problems with non-separable boundary conditions. Mosk. Univ. Publ., Moscow (2009). (in Russian).

6. M.A. Naimark. Linear differential operators. Nauka, Moscow (1969). [Part I: Elementary theory of linear differential operators, Frederick Ungar Publ. Co., New York (1967); Part II: Linear differential operators in Hilbert space, Frederick Ungar Publ. Co., New York (1968)].

7. B.E. Kanguzhin, G. Dairbaeva, Zh. Madibajuly. Unique recovering of boundary conditions for differential operator by set of spectra // Vestnik KazNU. Ser. Matem. 4 (104), 44-49 (2019). (in Russian).

8. B.E. Kanguzhin, G. Dairbaeva, Zh. Madibajuly. Identification of boundary conditions for differential operator // Vestnik KazNU. Ser. Matem. (103), 82-93 (2019). (in Russian).

Baltabek Esmatovich Kanguzhin,

Al-Farabi Kazakh National University,

Al-Farabi av. 71,

050040, Almaty, Kazakhstan

E-mail: kanbalta@mail.ru 